\title{
Competencia del estudiante de medicina para respetar las creencias y la cultura del paciente
}

\author{
Jorge Soler-González, Antoni Rodríguez-Rosich, Josep Ramon Marsal-Mora, en representación del grupo AP-UDL
}

Introducción. Las desigualdades en salud son injustas, innecesarias y evitables. La etapa de educación médica del grado es una oportunidad ideal para reflexionar en las prácticas y poder desarrollar habilidades y actitudes médicas basadas en el respeto, la equidad y la ecuanimidad de los futuros profesionales de la salud. Aprovechando la mejora del plan de estudios de nuestra facultad, diseñamos el presente estudio para comprobar si los estudiantes de medicina propondrían diferentes enfoques de la gestión del mismo hipotético caso clínico en función de la etnia del paciente.

Sujetos y métodos. Primera etapa: comparación de las respuestas de los dos grupos de alumnos a un cuestionario sobre un caso clínico hipotético; la única variable discriminatoria entre los grupos fue la fotografía del paciente que se adjuntó: un joven negro en un caso, y un joven blanco, en el otro. Segunda etapa: el trabajo en casa y en una sesión de feedback. Tercera etapa: en grupos pequeños se realizó un taller centrado en el tema con actividades de sensibilización del problema.

Resultados. Cuando el paciente era negro, se solicitaban un menor número de pruebas, el paciente tenía menos probabilidades de ser enviado a un hospital y había una percepción de que tendía a exagerar los síntomas para obtener un beneficio personal.

Conclusiones. Se encuentran diferencias en el tratamiento del paciente por los estudiantes de medicina. Estamos a favor de la incorporación de los conceptos de las desigualdades en salud y la competencia cultural en los estudios de medicina, con el fin de minimizar el efecto de estas ideas preconcebidas desde el inicio de la formación de los futuros profesionales de la salud.

Palabras clave. Aprendizaje basado en problemas. Educación de pregrado. Facultad de Medicina. Medicina familiar y comunitaria.

\section{Racial stereotyping and cultural competence: improving medical education}

Introduction. Health inequalities are multiple and complex and they are avoidable. Educating Medical student training is an ideal opportunity for reflecting on practices and for engendering skills and attitudes of respect, equity and equanimity among future health professionals. Coinciding with a modification of the teaching program at our faculty, we designed the present study to ascertain whether second-year medical students might propose different approaches to the management of the same hypothetical clinical case depending on the patient's ethnicity.

Subjects and methods. 1st stage: comparison of the responses of two groups of students to a questionnaire regarding a hypothetical clinical case. The only discriminatory variable between the groups was the photograph of the patient attached: a black youth in one case, and a white youth in the other. 2nd stage: home work and in a feedback session. 3rd stage: small-group workshop focused on the need for retraining and activities to heighten awareness of the problem.

Results. When the patient was black, fewer tests were requested, the patient was less likely to be sent to hospital, and there was a perception that he tended to exaggerate the symptoms to obtain personal benefit.

Conclusions. We found prejudice in patient management at the beginning of the medicine degree. We advocate the incorporation of concepts of health inequalities and cultural competence in medical studies, so as to minimize the effect of these preconceived ideas from the very beginning of the training of future health professionals.

Key words. Faculty of Medicine. Family and community medicine. Problem-based learning. Undergraduate education.
Facultad de Medicina; Universitat de Lleida. Atención Primaria; Institut Català de la Salut. Lleida, España.

Correspondencia:

Dr. Jorge Soler González. Facultad de Medicina. Universitat de Lleida. Montserrat Roig, 2. E-25008 Lleida.

E-mail:

jorgesolergonzalez@gmail.com

Agradecimientos:

A la Dra. Helen Minnis, lectora sénior de la Universidad de Glasgow, que ofreció sus cuestionarios y revisó el manuscrito.

Conflicto de intereses: No declarado.

Competing interests: None declared.

(c) 2014 FEM 


\section{Introducción}

En el desarrollo curricular del estudiante de medicina, el papel del médico de familia es esencial para aportar un concepto integrador en un magma de conocimientos científico-técnicos de múltiples disciplinas y competencias. Dentro de las competencias asignadas en el programa universitario para la formación de licenciados en medicina con el nuevo Plan de Bolonia, la Orden Ministerial ECI/332/2008, de 13 de febrero, incluye la competencia específica que hace referencia a la necesidad de que el alumno sea competente en saber abordar la práctica profesional respetando la autonomía del paciente, sus creencias y su cultura (competencia 46) [1]. Hasta la fecha, sabíamos bastante sobre el impacto que ha tenido el gran aumento de la inmigración en Europa incrementando las inequidades de salud. Por ejemplo, debido a ese inesperado crecimiento demográfico que ha sucedido en España, en el caso de los pacientes inmigrantes se han publicado varios artículos que muestran claras desigualdades en el uso de los servicios sanitarios $[2,3]$, de farmacia $[4,5]$ y de bajas laborales [6]. Las causas de su existencia son múltiples y complejas, y abarcan aspectos sociales, culturales, políticos, etc., pero no podemos negar que en muchos de los casos estas diferencias sanitarias son causadas por el propio sistema sanitario y por prejuicios de los profesionales que trabajan en él [7].

En el año 2001, Minnis et al [8] publicaron en British Medical Journal un estudio que demostraba que existían claras diferencias en el manejo clínico, la opinión y el enfoque de la entrevista clínica entre diversos grupos de psiquiatras que, valorando un idéntico caso clínico, se personalizaba con fotografías de supuestos pacientes de etnias diferentes.

Estas circunstancias han sido establecidas en algunos estudios con médicos especialistas, pero hasta la fecha no existen datos que evalúen si los alumnos de medicina ya se ven influidos por estos prejuicios. Sin embargo, sí se apunta la necesidad imperiosa de aumentar los conocimientos de pregrado en competencia cultural [9-12], siendo éste el momento idóneo para motivar y fomentar las reflexiones que condicionarán las habilidades y actitudes de respeto, equidad y ecuanimidad de los futuros profesionales médicos.

Aprovechando el cambio en el programa docente universitario de nuestra facultad [13], quisimos evaluar si había diferencias en el manejo de un mismo caso clínico por parte de los estudiantes de segundo curso de medicina.

\section{Sujetos y métodos}

Los autores, en calidad de profesores de la Facultad de Medicina de la Universitat de Lleida y médicos en ejercicio en atención primaria, nos proponemos abordar el tema de las desigualdades y la equidad en términos de salud y atención a la población inmigrante y de otras razas. El ámbito y la población diana de la propuesta será el personal en formación de pregrado, estudiantes de segundo curso de la Facultad de Medicina.

Los objetivos planteados en esta propuesta son tres:

- Conocer si existen diferencias en la valoración y toma de decisiones en relación con un caso clínico en función de la raza del paciente.

- Propiciar y motivar un proceso de feedback reflexivo en torno a los resultados del estudio y las características de igualdad, equidad, prejuicios y estereotipos en la prestación de servicios de salud.

- Abrir un campo docente para la reeducación y la toma de conciencia de la realidad en el campo de las desigualdades sanitarias.

La metodología utilizada se enmarca en tres niveles de actuación:

\section{Primer nivel}

Realización de un estudio comparativo entre dos grupos de estudiantes respondiendo el cuestionario sobre un mismo caso clínico. La única variable discriminante entre los grupos correspondía a la fotografía de un individuo de diferente raza como supuesto paciente.

La metodología de estudio propuesta por Minnis et al [8] nos pareció adecuada y atractiva para conseguir los objetivos. Se contactó con la revista British Medical Journal con el fin de solicitar permiso para utilizar su cuestionario y sus imágenes, y de igual forma se hizo con los autores del artículo. Tras obtener su autorización, y con la información recibida, se adaptó un borrador que valoraron los profesores de la asignatura para asegurar la relevancia y comprensión de las diferentes preguntas. A partir de los comentarios y sugerencias aportadas se elaboró la versión definitiva, constituida por una introducción del caso clínico en el que se presentaban los datos de filiación del paciente (nombre, sexo, edad y fotografía), una breve descripción de la historia clínica que resumía un caso clínico de dolor cólico en el hipocondrio derecho, sin síntomas de gravedad y manejable clínicamente desde la atención primaria, y 17 preguntas cerradas puntuables 
en una escala lineal de $10 \mathrm{~cm}$, que se convirtió posteriormente en numérica.

El cuestionario exploraba la importancia de registrar, en el caso concreto, ciertos datos de la historia clínica (consumo de drogas y antecedentes penales), preguntas sobre la dificultad de gestión del problema médico (dificultad diagnóstica del caso, necesidad de darle una baja laboral, necesidad de ingreso hospitalario o si anticipaban problemas en la adhesión a la medicación), preguntas sobre las distintas probabilidades de algunos diagnósticos diferenciales posibles (cólico hepático, intoxicación por drogas, ansiedad, cáncer). Finalmente, una última parte exploraba qué tipo de medicación se utilizaría y qué pruebas se indicarían para su manejo (ecografía, TAC).

El cuestionario se pasó en clase dividiendo al grupo en dos mitades una vez estuvieron sentados. Se pasó el cuestionario proyectado en PowerPoint a la primera mitad con una hoja de respuestas individual anónima y posteriormente se cambió a los alumnos del segundo grupo, a los que se hizo esperar fuera de la clase. La única diferencia entre los casos clínicos presentados era que el primer caso tenía el nombre y la fotografía de un joven negro y que el segundo caso era un joven blanco, ambas obtenidas del estudio de Minnis et al [8].

Para evitar que las diferencias pudieran atribuirse a una expresión facial diferente o de vestimenta, decidimos utilizar las mismas del estudio previo que habían sido seleccionadas con mucho esmero para evitar justamente el efecto descrito.

Para cada pregunta se compararon las medias entre los cuestionarios de los pacientes de raza negra y blanca, mediante el test $U$ de Mann-Whitney.

\section{Segundo nivel}

Realización por cada estudiante de un ejercicio de feedback reflexivo en torno a los principales resultados del estudio y centrado en base a unas preguntas. A tal efecto, los alumnos tenían en su documentación el redactado del caso y ambas presentaciones con las diferencias fotográficas, así como los principales resultados del estudio comparativo ( $\mathrm{Ta}-$ bla I). La tarea consistió entonces en trabajar sobre las siguientes preguntas:

- ¿Qué hemos encontrado en nuestro trabajo? Reflexionemos sobre por qué se han presentado así los dos casos y los resultados de cada pregunta para los dos grupos.

- Si pensáis que hay diferencias, ¿por qué han pasado? ¿Era algo esperable lo que ha sucedido esta tarde en clase?
Tabla I. Primer nivel de actuación correspondiente a las principales respuestas del cuestionario según si el paciente era blanco o negro.

\begin{tabular}{llcc}
\hline & Blanco & Negro & $p$ \\
\hline ¿Crees que está buscando conseguir una baja laboral? & 4,07 & 4,91 & 0,178 \\
\hline ¿Crees que es de los que abusa del sistema sanitario? & 4,07 & 4,95 & 0,122 \\
\hline ¿Crees que se tomará la medicación que le prescribirás? & 7,11 & 5,80 & 0,005 \\
\hline ¿Le inyectarías un analgésico? & 7,63 & 6,91 & 0,154 \\
\hline ¿Le pedirías una ecografía? & 8,22 & 7,64 & 0,341 \\
\hline ¿Le preguntarías si necesita la baja laboral? & 6,44 & 5,90 & 0,483 \\
\hline
\end{tabular}

- ¿Qué dice la bibliografía al respecto? ¿Hay algún estudio similar?

\section{Tercer nivel}

Talleres en grupo reducido de estudiantes (20 alumnos) para comentar y profundizar en los resultados y argumentaciones de los ejercicios. Si la primera expectativa es valorar si existen en los estudiantes prejuicios discriminativos en la valoración de aspectos relacionados con la salud, la verdadera actividad docente sería la reeducación y la toma de conciencia de la realidad, con la prevención de actitudes relacionadas.

\section{Responsabilidades éticas y protección de personas y datos}

El estudio fue aprobado por el Comité Ético de la Fundación IDIAP Jordi Gol en Atención Primaria. Los autores del manuscrito certifican que cumplen los principios éticos de publicación. El consentimiento informado de cada alumno no se obtuvo porque se realizó dentro de la clase de la facultad y los resultados se trabajaron de forma anónima.

\section{Resultados}

El grupo total de estudiantes estaba formado por 49 alumnos que asistieron a la clase. La edad media del grupo era de 20,3 años y el 76\% eran mujeres. Los alumnos se distribuyeron en dos subgrupos indistintamente, aunque no aleatorizados, de los cuales 22 realizaron el cuestionario asignado al joven de raza blanca y 27 al de raza negra. 
Figura. Representación de las diferencias según su intervalo de confianza (95\%).

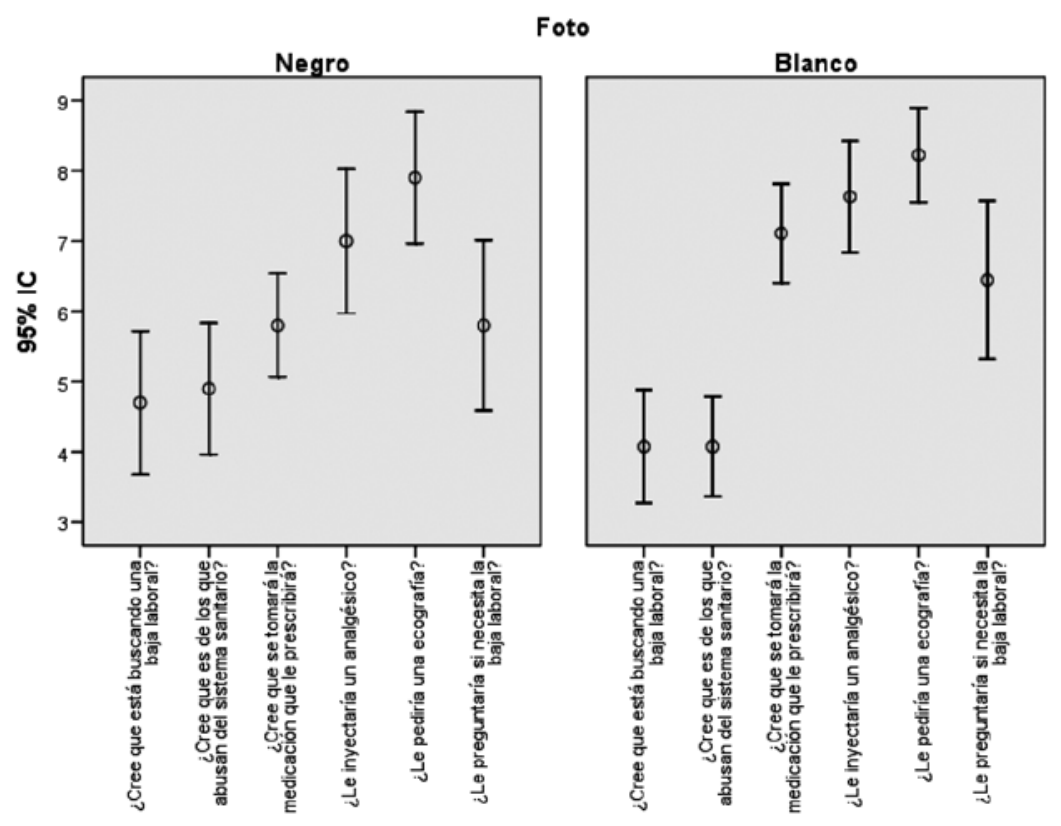

Tabla II. Segundo nivel: ejercicio de feedback con las 10 frases seleccionadas para reflexionar activamente en el taller extraídas de los trabajos realizados por algunos de los alumnos.

Creo que estas respuestas no eran esperables en una clase de medicina

Es posible que mis compañeros piensen que...

Es posible que algunos crean que...

Las diferencias son injustificables y espero que esto haya servido de reflexión

El estudio pierde valor por el pequeño tamaño de la muestra encuestada

Mi conclusión es que debemos ser muy precavidos a la hora de tomar en serio estos resultados

Las diferencias eran esperables porque aunque los grupos eran comparables, no eran iguales

Creo que se nos ha dado poco tiempo para responder las preguntas,

para no poder pensar ni responder razonadamente

Se puede concluir que hay una gran diferencia en el trato en el sistema sanitario

Algunos de los futuros médicos, a día de hoy y a pesar del momento social en el que se han criado y crecido, aún hacen diferencias en el trato que darían a un paciente de raza blanca y a otro de color

Las respuestas y la valoración en la escala lineal de 1 a $10 \mathrm{~cm}$ representan el grado de acuerdo o afirmación de la pregunta, con lo que queda esquema- tizada y distribuida según ambos subgrupos (Tabla I) (Figura).

En la segunda parte del trabajo se leyeron y valoraron los ejercicios individuales de reflexión personal. De todos ellos se extrajeron subjetivamente 10 frases para conducir la reflexión del tema a los talleres (Tabla II).

\section{Discusión}

En este trabajo hemos encontrado que nuestros alumnos de medicina priorizan de forma distinta algunos aspectos de la anamnesis según la raza del paciente. También hemos observado que cuando el caso parecía corresponder a un paciente negro, se solicitaban menos pruebas, había una menor probabilidad de enviarlo a un hospital y se percibía como un paciente con tendencia a exagerar los síntomas para conseguir beneficios personales.

En general, los resultados son comparables a los obtenidos por Minnis et al [8] al evaluar a especialistas en psiquiatría. En ambos estudios, las diferencias halladas en el manejo clínico son poco relevantes y ambos ponen en evidencia una cierta tendencia de prejuicios hacia determinados pacientes. Estos alumnos son jóvenes y han crecido y madurado en una sociedad multicultural, y comparten las aulas y los estudios con compañeros de diferentes países que cursan su licenciatura en la facultad. Sin embargo, las diferencias obtenidas indican un mejor trato hacia el paciente blanco.

Con nuestro trabajo añadimos una evidencia más hacia el conocimiento de que las desigualdades en salud existen y que conviven ya desde los primeros cursos de medicina. Son, sin duda, reflejo de prejuicios y estereotipos sociales trasladados al campo profesional. Todo apunta a que debemos trabajar con los alumnos desde un principio para evitar que estos prejuicios se consoliden y lleguen a generar problemas de asistencia en la vida profesional, promocionando estrategias de formación como las comunicadas en el ámbito de las emergencias médicas [14], la psiquiatría [15,16], la odontología [17], la traumatología [18], etc.

Diversas sociedades científicas han realizado un proceso de revisión y consenso con el fin de elaborar recomendaciones y directrices específicas para los programas que se centran en las necesidades curriculares de las desigualdades en salud [19]. Los objetivos de aprendizaje, contenidos, métodos de enseñanza y recursos útiles se han desarrollados principalmente para la enseñanza de los estudiantes de medicina, residentes y profesionales en la atención 
primaria. En el grado de Medicina se debe ayudar a los estudiantes a identificar y cultivar las actitudes que pueden ayudar a eliminar -en lugar de exacerbar potencialmente- las disparidades de salud. También se deben fomentar las habilidades de comunicación que permitan trabajar con pacientes de diversas culturas e idiomas para asegurar una alta calidad de la atención adecuada a cada paciente.

Utilizando las autorreflexiones expuestas posteriormente por los alumnos, consideramos que se han estimulado nuevas competencias y habilidades que fomentan el cambio de comportamiento, siendo los alumnos capaces de modular la influencia de las actitudes preexistentes en su comportamiento posterior.

Todo apunta a que los futuros programas de medicina incorporarán progresivamente propuestas docentes en esta línea para reducir los prejuicios raciales [20]. Sin embargo, hasta el momento son pocas las experiencias realizadas en las universidades y los conocimientos en competencia cultural que tienen los médicos residentes al acabar su formación, del mismo modo que son escasas las habilidades para ser competentes trabajando en sociedades multiculturales [21].

Ciertamente, como apuntaba una alumna, nuestro trabajo tiene como limitación el tamaño de la muestra, pero la intención era mostrar a los alumnos que las desigualdades en salud existen y que éstas nos implican sin que seamos conscientes de ello, incluso en el aula de una clase de medicina. Quizá aumentando el tamaño de la muestra de alumnos hubiéramos obtenido mayor significación estadística, pero no mayores razones para diseñar intervenciones formativas. Nos interesaba disponer de un material innovador que introdujera la metodología docente del modelo LEARN [22] y RESPECT [23, 24 , y que el alumno, con sus propias reflexiones, fuera consciente de la necesidad de adquirir competencias en ese campo. Otra de las limitaciones del trabajo presentado es que no evaluamos el resultado del impacto docente posterior a las clases realizadas (tercer objetivo docente), fundamentalmente porque tampoco era el objetivo del trabajo. Se ha descrito que simplemente una hora de clase sobre concienciación cultural conduce a diferencias entre los estudiantes asistentes [25,26]. Sin embargo, creemos muy interesante poder seguir la evolución de estos alumnos y realizar una evaluación que explore si estos alumnos tienen menos prejuicios que los alumnos que les preceden y que no han recibido esta formación.

En conclusión, hemos constatado que los prejuicios en el manejo del paciente existen ya desde los inicios de la carrera de medicina y, en nuestra opinión, es fundamental incorporar los conceptos de desigualdades en salud y competencia cultural en los estudios de medicina para evitarlos desde un principio.

\section{Bibliografía}

1. Orden Ministerial ECI/332/2008, de 13 de febrero de 2008 . Boletín Oficial del Estado. URL: http://www.boe.es/boe/ dias/2008/02/15/pdfs/A08351-08355.pdf.

2. Gimeno-Feliu LA, Macipe-Costa RM, Dolsac I, MagallónBotaya R, Luzón L, Prados-Torres A, et al. Frequency of attending primary care clinics by the immigrant versus autochthonous population. Aten Primaria 2011; 43: 544-50.

3. Soler-González J, Serna-Arnáiz C, Rué-Monné M, BoschGaya A, Ruiz-Magaz MC, Gervilla-Caño J. Utilización de recursos de asistencia primaria por parte de inmigrantes y autóctonos que han contactado con los servicios asistenciales de la ciudad de Lleida. Aten Primaria 2008; 40: 225-33.

4. Rué M, Bosch A, Serna MC, Soler-González J, Galván L, Cruz I. Observational study on drug spending in the immigrant and the indigenous population in the Lleida (Catalonia) health region. Aten Primaria 2010; 42: 328-37.

5. Cruz I, Serna C, Real J, Rué M, Soler J, Galván L. Comparison of the consumption of antidepressants in the immigrant and native populations in a Spanish health region: an observational study. BMC Public Health 2010; 17: 255.

6. Soler-González J, Serna MC, Bosch A, Ruiz MC, Huertas E, Rué M. Sick leave among native and immigrant workers in Spain -a 6-month follow-up study. Scand J Work Environ Health 2008; 34: 438-43.

7. Lavizzo-Mourey R, Lumpkin JR. From unequal treatment to quality care. Ann Intern Med 2004; 141: 221.

8. Minnis H, McMillan A, Gillies M, Smith S. Racial stereotyping: survey of psychiatrists in the United Kingdom. BMJ 2001; 323: 905-6.

9. Geiger HJ. Racial stereotyping and medicine: the need for cultural competence. CMAJ 2001; 164: 1699-700.

10. Loudon RF, Anderson PM, Gill PS, Greenfield SM. Educating medical students for work in culturally diverse societies. JAMA 1999; 282: 875-80.

11. Betancourt JR, Cervantes MC. Cross-cultural medical education in the United States: key principles and experiences. Kaohsiung J Med Sci 2009; 25: 471-8.

12. Li BU, Caniano DA, Comer RC. A cultural diversity curriculum: combining didactic, problem-solving, and simulated experiences. J Am Med Womens Assoc 1998; 53 (Suppl 3): 128-30.

13. Soler-González J, Rodríguez-Rosich A, Ribera-Calvet J, Peñascal-Pujol E, Serna C, Martí RM, en representación del grupo AP-UDL. Medicina de Familia como asignatura obligatoria en el grado de Medicina de la Universitat de Lleida: análisis de la percepción de los estudiantes. FEM 2013; 16: 83-9.

14. Richardson LD, Babcock-Irvin C, Tamayo-Sarver JH. Racial and ethnic disparities in the clinical practice of emergency medicine. Acad Emerg Med 2003; 10: 1184-8.

15. Kales HC, Neighbors HW, Blow FC, Taylor KK, Gillon L, Welsh DE, et al. Race, gender, and psychiatrists' diagnosis and treatment of major depression among elderly patients. Psychiatr Serv 2005; 56: 721-8.

16. Conner KO, Copeland VC, Grote NK, Koeske G, Rosen D, Reynolds CF 3rd, et al. Mental health treatment seeking among older adults with depression: the impact of stigma and race. Am J Geriatr Psychiatry 2010; 18: 531-43.

17. Gregorczyk SM, Bailit HL. Assessing the cultural competency of dental students and residents. J Dent Educ 2008; 72: 1122-7.

18. Dykes DC, White AA 3rd. Getting to equal: strategies to understand and eliminate general and orthopaedic healthcare disparities. Clin Orthop Relat Res 2009; 467: 2598-605.

19. Smith WR, Betancourt JR, Wynia MK, Bussey-Jones J, Stone VE, Phillips CO. Recommendations for teaching 
about racial and ethnic disparities in health and health care. Ann Intern Med 2007; 147: 654-65.

20. Burgess D, Van Ryn M, Dovidio J, Saha S. Reducing racial bias among health care providers: lessons from socialcognitive psychology. J Gen Intern Med 2007; 22: 882-7.

21. Park ER, Betancourt JR, Kim MK, Maina AW, Blumenthal D, Weissman JS. Mixed messages: residents' experiences learning cross-cultural care. Acad Med 2005; 80: 874-80.

22. Berlin E, Fowkes W. A teaching framework for cross-cultural health care. Western J Med 1983; 139: 934-8.

23. Kleinman A, Eisenberg L, Good B. Culture, illness, and care: clinical lessons from anthropologic and cross-cultural research. Ann Intern Med 1978; 88: 251-8.

24. Mostow C, Crosson J, Gordon S, Chapman S, González P, Hardt E, et al. Treating and precepting with RESPECT: a relational model addressing race, ethnicity, and culture in medical training. J Gen Intern Med 2010; 25: S146-54

25. Walton J. Can a one-hour presentation make an impact on cultural awareness? Nephrol Nurs J 2011; 38: 21-30.

26. Abbey JH. The impact of education and experience on physician assistant students' perception of cultural awareness, knowledge and skills. J Allied Health 2006; 35: e24-36. 